\title{
Application of Sonar Equation in the Design Of Ocean Instruments
}

\author{
Ying Yang 1,2,3, Li Hui 1,2,3,a, Xiangtao Ran 1,2,3, Min Liu 1,2,3 ${ }^{\text {Li Yang }}{ }^{1,2,3}$, \\ Yang Zhou ${ }^{1,2,3}$
}

${ }^{1}$ Qilu University of Technology (Shandong Academy of Sciences), Institute of Oceanographic Instrumentation, Shandong Academy of Sciences, Qingdao 266000,china;

${ }^{2}$ Shandong Provincial Key Laboratory of Marine monitoring instrument equipment technology, Qing dao 266000 ,china;

${ }^{3}$ National Engineering and Technological Research Center of Marine Monitoring Equipment, Qingdao 266000,china.

a11932807@qq.com

\begin{abstract}
This paper introduces the principle of sonar and the basic equation of sonar, and the extensive application of sonar equation in marine instruments. By knowing the basic parameters of ultrasonic transducer, the parameters of the function of the instrument are calculated, such as the loss of sound wave propagation and the intensity of the target in the sonar equation. Finally, the minimum electric power required in the design of the marine instrument is calculated.
\end{abstract}

Keywords: sonar equation, current measurement, Doppler.

\section{Introduction}

Sonar can be divided into active sonar and passive sonar according to its working principle. Sounder, communication sonar, mine detection sonar, echo station, etc., belonging to active sonar. Noise station and reconnaissance sonar are classified as passive sonar.

Active sonar is a purposeful active emission of acoustic signals from a system. Because the target information is stored in the echo, therefore, the existence of the target can be judged by the received echo signal and the distance, azimuth, velocity and other parameters of the target can be estimated or calculated. A sounder, a current meter, etc., can infer the distance of the target by the time delay between the echo signal and the transmitting signal, and the direction of the target can also be inferred by the principle of the normal line in front of the echo wave. The deviation of the frequency between the transmitted signal and the echo signal can deduce the radial velocity of the target.

Active sonar is composed of transceiver array, transceiver conversion, transmitter, receiver, controller, monitor and so on. As shown in Fig.1.

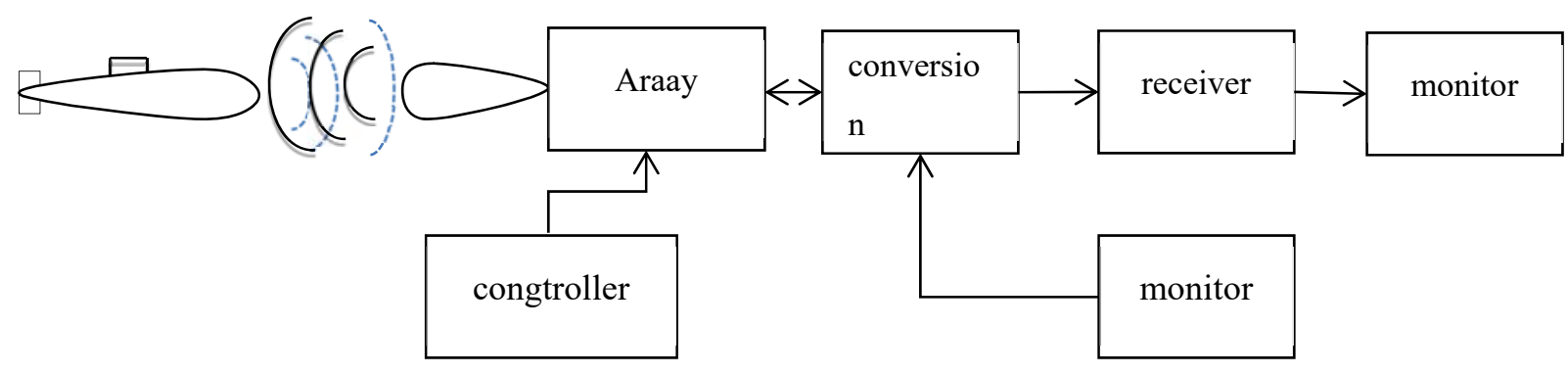

Fig.1 Sonar principle block diagram

The use of transducer array is to gather the sound energy into a predetermined direction to form a beam, transmit the acoustic signal through the transducer, propagate the acoustic signal through the sea water, meet the reflection of the target, and receive the echo signal and noise by each array element of the transducer array, and convert the sound signal into the electrical signal through the transceiver conversion circuit. The active sonar initiatively launches the detection signal, and can measure the 
distance of the precise target by the time difference between the transmitting and receiving signals. The echo sounder, the current meter and the wave meter are the principles of this active sonar.

\section{Sonar Equation}

Sonar equation is the relation between environment, propagation medium, measurement target and various parameters of equipment. One of the functions of sonar equations is to predict the performance of sonar devices that have been designed and are being designed, and to estimate the performance of some parameters such as detection probability and search probability. Another function is sonar design. This paper introduces the application of sonar equation. The measurement of sea depth, current, wave and other parameters is very important for marine environmental monitoring. How to design a good instrument to measure these parameters, such as sounder, current meter, wave meter and so on, sonar design is particularly important in the design and development of equipment.

\subsection{Active Sonar Basic Equation}

Because the principle of sounder, current meter, wave meter and so on belongs to active sonar, according to the working process of active sonar, the equation of active sonar can be written conveniently.

In this paper, we consider an active sonar with a transmitter and transceiver, which has a radiating sound source level SL, and the receiver directivity index is DI. The propagation loss from the sound source to the target is TL, the target intensity is TS, the detection threshold of the space-time processor is DT, and the background interference is ambient noise. The sound level is NL within the operating bandwidth of the device. Due to the loss of sound propagation, when the sound level of the sound signal at the source level SL reaches the target, the sound level is reduced to SL-TL. Because the target intensity is TS, from the definition of TS, the sound level at the unit distance from the center of the target is SL-TL+TS in the return direction, and the sound level of the echo arriving at the receiving array is SL-2TL+TS, which is usually called echo signal level. On the other hand, the background noise acts on the receiver transducer, but it is suppressed by the receiver directivity of the receiving array, and the noise level of the interference is NL-DI. Because the transducer's acoustic axis always points to the target, the echo level is not depressed by the reception directivity. The echo signal and noise are converted by the transducer to an electrical signal and sent to the processor, the signal-to-noise ratio of the signal (in decibels) is:

$$
(\mathrm{SL}-2 \mathrm{TL}+\mathrm{TS})-(\mathrm{NL}-\mathrm{DI})
$$

If this signal-to-noise ratio is greater than the detection threshold DT of the receiver, the target can be detected by the system. When the signal / noise ratio is exactly equal to the detection threshold, the system is just detectable. The active sonar equation with noise as the main background interference can be obtained by using the detection threshold.

$$
\mathrm{SL}-2 \mathrm{TL}+\mathrm{TS}-(\mathrm{NL}-\mathrm{DI})=\mathrm{DT}
$$

The sound source level is defined as the decibel number of the ratio of sound source intensity to reference intensity at $1 \mathrm{~m}$ along the sound axis, that is,

$$
S L=10 \lg ^{\text {strength of sound source }} / \text { sonar reference intensity }
$$

The reference intensity is the effective value of sound pressure $1 \mu \mathrm{Pa}$. The sound source level of the active sonar is mainly determined by the axial sound power of the transducer. Its relation to the electric power $\mathrm{Pa}$ added to the transducer, the electro-acoustic conversion efficiency $\eta$ and the emission directivity index or the aggregation coefficient $\mathrm{DI}_{\mathrm{T}}$ of the transducer are as follows:

$$
\mathrm{SL}=170.8+10 \log (\eta \mathrm{Pa})+\mathrm{DI}_{\mathrm{T}}
$$




\section{The Significance of Current Measurement}

The velocity and direction of the ocean can represent the macroscopic flow of sea water. Ocean currents play an important role in energy, heat, mass transport and pollutant drift within the ocean, connecting the world's oceans like the human body's blood circulation. The current actually acts as a heat exchange in the seawater. In the process of moving from one sea area to another, the current can move the high-temperature sea water to the lower-temperature sea area, thus warming the sea surface air. On the other hand, sea water with low temperature can be moved to the waters with higher temperature, which makes the sea surface air temperature drop, and thus plays a role in regulating the climate. From the surface to the bottom of the ocean, there are currents at the sea, whose spatial and temporal scales are continuous, but their velocities vary from sea to sea. The physical and chemical properties and biological characteristics of sea water are different in different sea areas, and there are different plankton and various kinds of fish, and many fish also migrate with the current. In addition, with the development of society and the improvement of science and technology, the data of ocean waves, tides and currents are needed in China's marine resources survey, offshore oil platform and offshore engineering construction, port wharf design, military equipment research, and so on. In addition, according to incomplete statistics, China's direct economic losses due to marine disasters are relatively large.

\section{The Principle of Acoustic Doppler Current Measurement}

When the transducer is working, a part of the energy is reflected back to the transducer due to the scattering body in the water during the propagation of the acoustic signal. At the time of measurement, assuming that the velocity of water in a horizontal plane is uniform, then in the range of measurement, the water in the beam moves along the beam, and the velocity of motion is $V_{b i}(n)$, According to the Doppler principle, because of this motion, there is a Doppler frequency offset between the echo frequency of the beam and the frequency of the acoustic wave. The relationship between the Doppler frequency offset and the velocity of the water body can be expressed as follows:

$$
v_{b}(n)=\frac{c f_{d}(n)}{2 f}
$$

In the equality, fd represents frequency offset, while $\mathrm{n}$ denotes $\mathrm{n}$ layer, $\mathrm{f}$ is the frequency of sonic wave emission. $\operatorname{Vbi}(\mathrm{n})$ denotes the projection of the velocity of the $\mathrm{n}$ layer water body on the $\mathrm{i}$ beam, which is a scalar.

There are a large number of acoustic scatterers in the sea water. When the acoustic waves with frequency f0 propagate in the sea water, some of the energy is scattered back by these scatterers and received by the transducer, and the frequency is measured to be fr after amplification. According to the principle of Doppler frequency shift, as long as there is a relative motion between the source (or receiver) and the scatterer, then $\mathrm{fr} \neq \mathrm{f} 0$, the difference is $\mathrm{fd}$. The formula is:

$$
f_{d}=f_{r}-f_{0}=2 f_{0} V \sin \alpha / c
$$

Where $\alpha$ is the vertical angle of the beam, $\mathrm{V}$ is the horizontal relative velocity of the sound source (or receiver) and the measured scatterers, $\mathrm{c}$ is a velocity of sound.

When the sound wave is reflected, the scattering echo time returned from different depth layers is different. The relative velocities of scatterers in different layers can be obtained by detecting the frequency shifts at different times.

As shown in the Fig.2, a sound wave is emitted at time 0, which is in the form of an acoustic pulse, assuming that the width of the sound pulse is $\mathrm{T}$. The sound wave propagates along the ray bi in the water body. After $\mathrm{tn} / 2$, the initial end of the acoustic pulse reaches the zn layer, so the distance 
between the $\mathrm{zn}$ layer and the instrument is the distance of the sound wave propagation during this period of time. As a result of the scattering action of the scatterers, a portion of the energy returns along the ray and passes through tn $/ 2$ to reach the transducer. The acoustic wave transmission energy continues to propagate forward, $\mathrm{tn} / 2+\mathrm{T}$, the beginning of the acoustic pulse reaches the $\mathrm{zn}+1$ layer, the end of the acoustic pulse reaches the zn layer, and after $\mathrm{tn} / 2$, the acoustic wave pulse ends reach the transducer. If the signal is received from the tn moment to the end of $\mathrm{tn}+\mathrm{T}$, the echo received during this period is the echo of the acoustic pulse scattered in the zn layer, and the Doppler shift of the echo signal reflects the velocity of the water body in the zn layer. Based on the time scale, the velocity meter can collect the echo from different layers in space, and then complete the velocity measurement of each layer. Then according to the principle of vector synthesis, the velocity vector of each layer of water can be obtained.

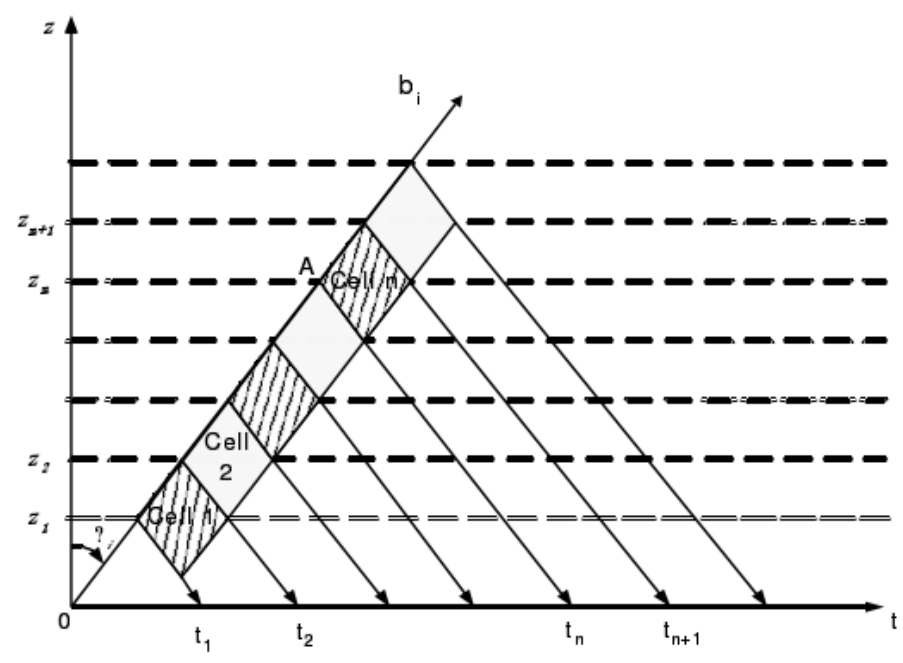

Fig.2 Echo layering diagram

\section{Design Example}

Apply sonar equation to the design of ocean current meter. If a current meter is designed to work at $400 \mathrm{KHz}$, the basic parameters of the current meter, such as basic depth, sonar emission frequency, beam width, and other parameters.

The propagation loss is derived from the spherical propagation loss plus the absorption loss, as follows:

$$
P L=20 \lg r+\alpha r \times 10^{3}(d B)
$$

The viscosity of water, the chemical relaxation process of solutes such as magnesium sulfate and boric acid result in the absorption of acoustic energy by sea water when acoustic waves propagate in seawater. The absorption coefficient of sea water is related to the frequency of sound wave, sea water temperature, salt and static pressure, etc. The lower the frequency of sound wave is, the smaller the absorption coefficient is. In the design, the frequency of sound wave reaches $400 \mathrm{kHz}$, and the acoustic absorption of seawater is caused by the viscosity of water. The approximate formula for the absorption coefficient $\alpha$ of seawater under standard conditions is as follows:

$$
\alpha=\frac{0.1 \times f^{2}}{1+f^{2}}+\frac{40 \times f^{2}}{4.1+f^{2}}+\frac{2.75 \times f^{2}}{10000}+0.03=84.1 \mathrm{~dB} / \mathrm{km}
$$


Typically, 1.0936 multiples are selected as absorption coefficient values, as follows:1.0936 $\times$ $84.1=91.97 \mathrm{~dB} / \mathrm{km}$, The propagation loss of seawater under standard conditions when $\mathrm{r}=100 \mathrm{~m}$ is:

$$
\mathrm{PL}=20 \lg r+\alpha \mathrm{r} \times 10^{-3}(\mathrm{~dB})=49 \mathrm{~dB}
$$

The solid angle of $5^{\circ}$ will be generated by a rectangular array with L side length or a ring array with a larger diameter. Therefore, the equivalent diameter $\mathrm{D}$ of the transducer is $0.084 \mathrm{~m}$ from the directivity diagram of the transducer.

The directivity index DI for launching and receiving is:

$$
\mathrm{DI}=10 \lg \left(\frac{\pi \mathrm{D}}{\lambda}\right)^{2}=10 \lg \left(\frac{3.14 \times 0.084}{0.00375}\right)^{2}=36.9(\mathrm{~dB})
$$

Next, the sound source level SL is obtained from the active sonar equation under the background of noise. The precondition is to know the target strength TS. In the case of vertical incidence, sea level is an extremely strong target of reflection and its intensity is quite clear, but the current measurement is a measurement of the velocity of the sea profile, resulting in volume reverberation through the inhomogeneous structure of marine life and non-organisms, as well as the seawater itself. From this we need to know the scattering intensity of sea water. The scattering intensity is a basic ratio representing reverberation, which is equal to the ratio of the sound intensity scattered by unit area or volume at a reference distance of 1 meter to the intensity of incident plane wave in decibels. In order to determine the strength of reverberation volume TS, it is necessary to calculate $10 \operatorname{lgV}$. The volume of scattering intensity depends on the beam width of the transmitting and receiving transducers:

$$
V=\frac{c T}{2} \frac{\pi R^{2} \theta_{h} \theta_{V}}{4}
$$

The equivalent beam width $\theta_{h}$ and $\theta_{V}$ in the horizontal and vertical directions represented by radians is derived from the known depth and the incident angle of the transducer.

$$
V=\frac{1500 \times 1333 \times 10^{-6}}{2} \times 3.14 \times \frac{(0.044 \times 100)^{2}}{4}=15.45 \mathrm{~m}^{3}
$$

Scattering intensity active sonar equation:

$$
T S=S_{S, V}+10 \lg A, V
$$

Under the condition of vertical incidence, the incident intensity of seabed is $10 \mathrm{~dB}$, the target intensity of single fish is $-50 \mathrm{~dB}$, and the intensity of sea water scattering is about $-90 \mathrm{~dB}$.

$$
\mathrm{TS}=\mathrm{S}_{\mathrm{S}, \mathrm{V}}+10 \lg \mathrm{A}, \mathrm{V}=-78.11 \mathrm{~dB}
$$

The noise of the ocean itself is also the environmental noise, the self - noise and the noise caused by the sonar receiver can not be ignored, and the ambient noise refers to the noise which is transmitted to the transducer from all directions around, and the ambient noise level acts as a sonar parameter, which is expressed in decibels by the sound intensity of the ambient noise measured by the transducer. 


$$
N L_{0}=-15+20 \lg f=37 d B
$$

The self-noise can be seen as zero when the transducer is designed. The sonar receiver noise through the transmission bandwidth of the transducer can be obtained to be about $28.75 \mathrm{~dB}$. From this, the noise intensity $\mathrm{NL}=37+28.75=65.75 \mathrm{~dB}$ is obtained.

The active sonar equation is:

$$
2 \mathrm{PL}=\mathrm{SL}+\mathrm{TS}-\mathrm{N}+\mathrm{DI}-\mathrm{DT}
$$

So there is:

$$
\mathrm{SL}=2 \mathrm{PL}-\mathrm{TS}+\mathrm{N}-\mathrm{DI}+\mathrm{DT}
$$

The sound source level at a depth of $100 \mathrm{~m}$ is: $204.96(\mathrm{~dB})$. Therefore, the maximum sound source level is $205 \mathrm{~dB}$.

$$
S L=10 \lg P+170.8+D I_{t}
$$

So:

$$
101 \text { g } P=205-170.8-36.9=-2.7(\mathrm{~dB})
$$

Electric power $\mathrm{P}=0.53 \mathrm{~W}$.

\section{Conclusion}

In fact, it is very easy to design a sonar transmitter with electrical power greater than $0.53 \mathrm{~W}$. The current meter, the sounder and the wave meter are all based on the measurement of water depth, and the target intensity of the sounder and the wave detector is corresponding to the sea bottom or sea surface intensity, which is less difficult than that of the current meter.

\section{Acknowledgments}

This work is supported by Production of Acoustic Wave and Tide Gauge based on underwater platform (granted No. 2016YFC1400403), Research and demonstration on Integrated Technology of Long-Term Integrated observation system for Ecological Environment of Ocean Station (granted No. 201505007-3) and National Marine Monitoring equipment Engineering Technology Research Center (granted No. 2016GGH4501).

\section{References}

[1]. Igor Prokhorov,Andrei Sushchenko,Vladimir Kan,Evgeny Kovalenko. Simulation of Sonar Signal Propagation in a Fluctuating Ocean[J]. Physics Procedia,2015,70.

[2]. Acoust. Urick R J. J. Society of America . 1962.

[3]. Antonio Ruiz-Mayor,Juan-Carlos Crespo,Gracian Trivino. Perceptual ambiguity maps for robot localizability with range perception[J]. Expert Systems With Applications,2017,85.

[4]. Some consequence of stochastic sonar equation in underwater warfare Assessment. Jean-Yves Morin,,Pierre-Yves Arques. 1993. 
[5]. The RRA algorithm:recursive ray acoustics forthree-dimensional speeds of sound. ZIOMEK L J. IEEE,Oceanic Engineering . 1993.

[6]. Receiver depth selection for passive Sonar systems. Ferla C.M,,Porter M.B. IEEE Journal of Oceanic Engineering . 1991.

[7]. Acoust. Wilson G R,Koch R A,Vidmar P J. J. Society of America . 1988.

[8]. Receiver depth selection for passive sonar systems. Ferla, Carlo,Porter, Michael B. IEEE Journal of Oceanic Engineering . 1991.

[9]. P.G. Auran,O. Silven. Underwater sonar range sensing and $3 \mathrm{~d}$ image formation[J]. Control Engineering Practice, 1996,4(3).

[10]. C.S. Tong,Y.W. Yeung. Boundary location from range data using Hough transform[J]. Pattern Recognition,2001,34(10).

[11]. Heiko Bülow,Max Pfingsthorn,Andreas Birk. Using Robust Spectral Registration for Scan Matching of Sonar Range Data[J]. IFAC Proceedings Volumes,2010,43(16).

[12]. Choudhury Maroof H,Barreto Armando. Design of a multi-sensor sonar system for indoor range measurement as a navigational aid for the blind.[J]. Biomedical Sciences Instrumentation, 2003,39 . 